\title{
MEASUREMENTS OF STRAIN FIELDS DUE TO NANOSCALE PRECIPITATES USING THE PHASE IMAGE METHOD
}

\author{
PATRICIA DONNADIEU ${ }^{1}$, KENJI MATSUDA ${ }^{2}$, THIERRY EPICIER ${ }^{3}$ AND JOEL DOUIN $^{4}$ \\ ${ }^{1}$ LTPCM-ENSEEG-INPG-Domaine Universitaire - BP 75-F38402 St Martin d'Hères, France, ${ }^{2}$ Department of \\ Materials Science and Engineering, Toyama University 3190 Gofuku, 930-8555, Japan, ${ }^{3}$ GEMPPM, INSA- \\ LYON, Bat 502, 20, av. Einstein, F69621 Villeurbanne, France, ${ }^{4}$ LEM CNRS-ONERA, 29 av. Division \\ Leclerc, F92322 Chatillon, France \\ e-mail: donnadie@ltpcm.inpg.fr \\ (Accepted August 28, 2001)
}

\begin{abstract}
Owing the phase image method (Hytch, 1998), strain fields can be derived from HREM images. The method is here applied to the nanoscale precipitates responsible for hardening in Aluminum alloys. Since the method is a very sensitive one, we have examined the impact of several aspects of the image quality (noise, fluctuations, distortion). The strain field information derived from the HREM image analysis is further introduced in a simulation of the dislocation motion in the matrix.
\end{abstract}

Keywords: displacement field, HREM, phase image.

\section{INTRODUCTION}

Nanoscale precipitation is frequently responsible for strengthening. In Aluminum alloys, the microstructure typical of age hardening is a large dispersion of nanometric precipitates (density $\approx 10^{15}-10^{17} / \mathrm{cm}^{3}$, size 1 $50 \mathrm{~nm}$ ). In such microstructure, the dislocation motion is mainly affected by the precipitate structure and the stress fields they create in the matrix. The former effect controls the possibilities for the dislocations to shear the precipitates while the latter concerns propagation of dislocations within the matrix. Since hardening precipitation often occurs by decomposition of a supersaturated solution, the precipitates do not belong to the phase diagram. Besides, the small precipitate size makes direct determination of composition and structure difficult. Thus there is no easy way to know the stress and strain fields around the precipitates. Therefore, it is of interest to develop a method to measure them.

\section{MATERIALS AND METHOD}

The phase image method proposed by Hytch et al. (1998) allows extracting quantitative information on atomic displacements from high resolution electron microscopy (HREM) images. The method is based on the ability of HREM to provide an atomic resolution projection of the structure. The image of a crystalline area can be decomposed in a set of periodic components g. In a perfect lattice, the phase of a g- component is constant, while in a lattice distorted by a displacement field $\mathrm{u}(\mathrm{x}, \mathrm{y})$, the phase is $-2 \pi \mathrm{g} \cdot \mathrm{u}(\mathrm{x}, \mathrm{y})$. This property results of image formation in HREM. In each point of the phase map, the intensity is proportional to the displacement $\mathrm{u}(\mathrm{x}, \mathrm{y})$ (scaling factor: 256 grey levels for a fringe spacing $2 \pi / g$ ).

The phase method is illustrated in Fig. 1 by a HREM image of a precipitate embedded in the $\mathrm{Al}$ matrix and the phase images for two Fourier components $g_{1}$ and $g_{2}$. Notice that since $g_{1}$ and $g_{2}$ are not colinear, this allows for a complete determination of the displacement field in the plane of the micrograph but no information on the component of $u$ perpendicular to the image. However, for precipitates elongated along one direction, which is hopefully quite frequent in hardening precipitation, the displacement field is very small, if not zero, along this direction, and thus the method leads to a complete determination of the displacement field. Finally, the strain and stress tensors components can be further derived from the displacement fields obtained using the usual equations of elasticity.

The method, which can be described as a numerical moiré, has high accuracy. This was exemplified by tests on simulated HREM images of a precipitate embedded in a matrix which strain field was calculated using a model from Mott and Nabarro (1940). Even when the strain field was decreased down a level too small to be detected by careful eye inspection, the phase images were still able to evidence the displacements (Douin et al., 2001). 


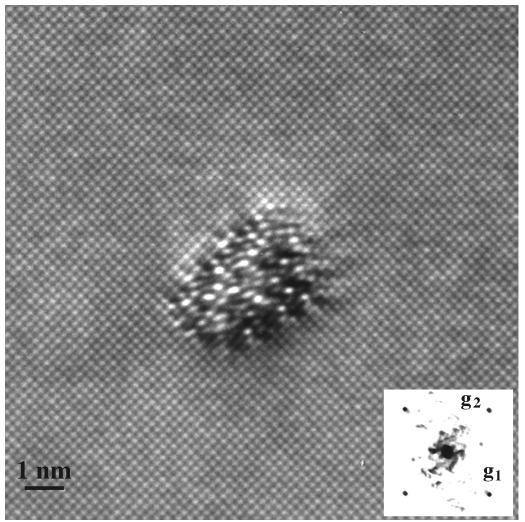

a)

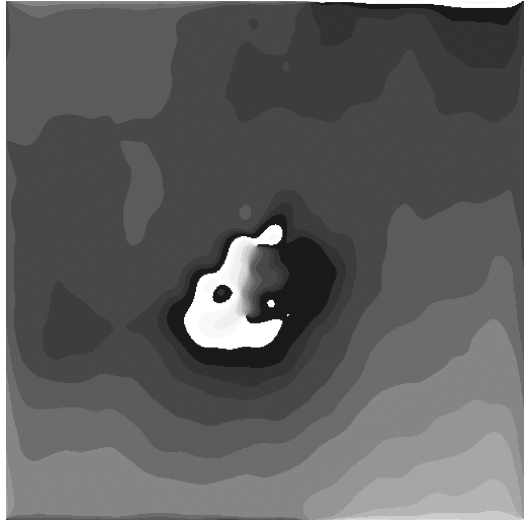

b)

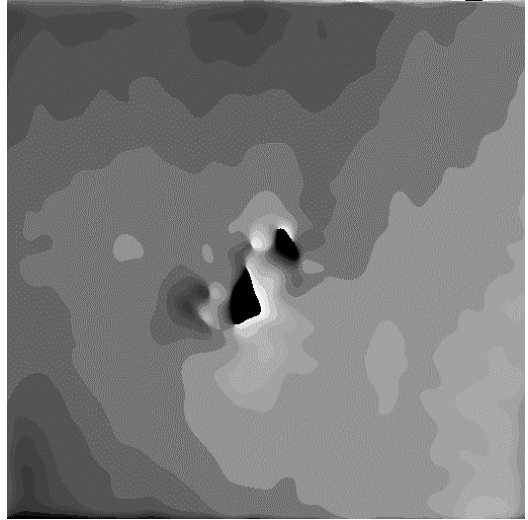

c)

Fig. 1. A HREM image and related phase images. a) HREM image of a precipitate embedded in the Al matrix. Two g-components of the Al lattice are indicated on the FFT in the insert. $b, c$ give the maps of displacements $u_{1}$ and $u_{2}$ for each component $g_{1}$ and $g_{2}$.

The system of interest here is a Al-Mg-Si alloy at peak hardening (namely a 6056 alloy in the T6 state). Our goal is to carry out a direct measurement of the displacement field and further use computer simulation of the dislocation motion in the stress field derived from the measured displacement field, but the present communication will emphasize on the measurements of displacement field. In the alloy of interest here, the precipitates are formed of complex phases and frequently have uneven shapes. Therefore, in such a case, only direct measurements can allow to evaluate the strain and stress fields and further the impact of irregular shape on dislocation propagation.

The phase images are here obtained following the method given by Hytch et al. (1998). The phase image technique being recent, its possibilities and limits remain to explore. In the present work, the measurement of the displacement field lead to specific difficulties: study of large areas (width in the order of 20 to $40 \mathrm{~nm}$ ) and small grey level signals. Because of these imposed conditions, we have to identify the impact of the image quality. The present work reports an empirical approach pointing out the difficulties and proposing some solutions.

\section{RESULTS}

\section{NOISE AT ATOMIC SCALE}

The drawback of the high sensitivity of the phase image method is that very small features can generate perturbations on the image. Since the HREM image contrast results from the interference of the electron beam going through the samples, noise may be generated by several local fluctuations: for instance, contamination, surface roughness, pollution surface layer resulting from sample preparation,. These noises may occur at the atomic scale as well as larger scale. Besides, the HREM images are made at high magnification and hence with a weak electron signal, which contributes to decrease the signal/noise ratio.

The phase image is obtained by the Fourier transform of an area in the reciprocal space selected by a mask centered on a $g$ component. A circular mask with gaussian edges having the radius equals to the size of the first Brillouin zone insures to keep all the distortion information. In principle, to get the displacement field requires to use a mask radius $g / 2$. Reducing the mask size has been tested here on several cases. Fig. 2 and 3 illustrate the effect of mask size reduction on experimental and simulated HREM images. The experimental image (Fig. 2) shows that a mask of radius $\mathrm{g} / 6$ reduces the atomic scale noise. On the other hand, the simulated HREM image allows to determine the minimum mask radius that can be used without altering the measurements. Fig. 3b, c compare the phase images obtained on a simulated image using a mask radius $\mathrm{g} / 2$ or $\mathrm{g} / 6$, respectively. Subtraction of images such as Fig. 3b, c indicates that a mask with a radius $\mathrm{g} / 6$ instead of $\mathrm{g} / 2$ removes details of amplitude $0.003 \mathrm{~nm}$ error at $2 \mathrm{~nm}$ from the precipitate center. At the precipitate/matrix interface, the mask effect is stronger but measurements in the very vicinity of the precipitates should be avoided because of an artifact specific of HREM image. Therefore, as far as the displacement field at the interface is not of interest, the mask size can be reduce up to $g / 6$ without significantly altering the displacement field measurements.

We interpret the small sensitivity to the mask radius reduction as a consequence of the different scale involved: the displacement field has a long- 
range influence compared to the noise scale. In terms of Brillouin zone, the long-range information corresponds to components close to the zone center, while the short-range information corresponds to reciprocal vectors close to the Brillouin zone boundaries. It is worth noting that the noise at atomic scale on phase images is a trouble commonly met in phase images applications. To eliminate this noise, some authors have used finite element approach (Kim, in preparation). Our study indicates that, as far as the information of interest is a quite long-range one, a mask size reduction can be an efficient solution to remove the atomic scale noise.

\section{LONG RANGE FLUCTUATIONS OF IMAGE INTENSITIES}

Measurements of the displacement field around precipitates require to study large area and to deal with quite weak signal since the total deformation is about $0.2 \mathrm{~nm}$ on a $40 \mathrm{~nm}$ large area. On such large areas, the HREM images can be spoiled in place by contamination layers and irradiation effects. For example, an electron beam creates damages in aluminum for acceleration voltage higher than $120 \mathrm{keV}$. The image in Fig. 1a taken on a transmission electron microscope Topcon $2 \mathrm{~B}$ is a particularly high quality one because this microscope is able to operate at $100 \mathrm{keV}$ with sufficient resolution to resolve the lattice distance $(0.2 \mathrm{~nm})$. Usually such a resolution needs higher voltage. Notice that contamination layers can be reduced by short ion milling prior to the HREM work but still contamination during observations is difficult to avoid.

Thickness variation across the area of interest may also alter the images. In the present case, the sample preparation (electropolishing) and composition ( $\mathrm{Al}, \mathrm{Mg}$ and $\mathrm{Si}$ ) do not give rise to preferential etching. However, on large areas, continuous thickness variation is possible. Assuming a wedge shape sample, the thickness variation can be estimated a few nanometers. In addition, we do not observe inversion of the column contrast in the studied areas, we consider thickness variation as having a negligible influence here.

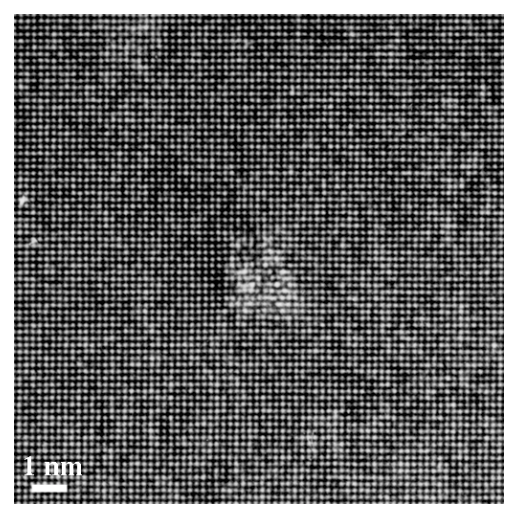

a)

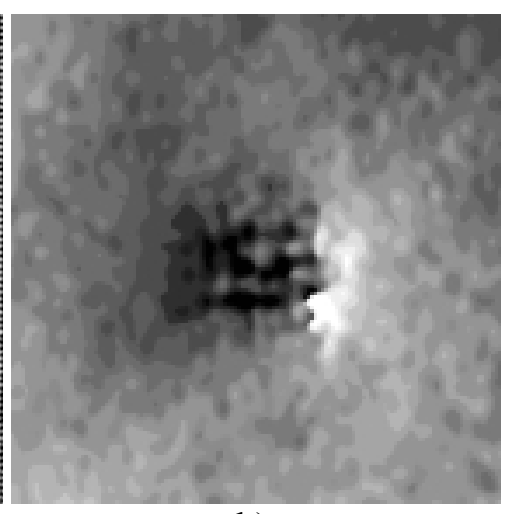

b)

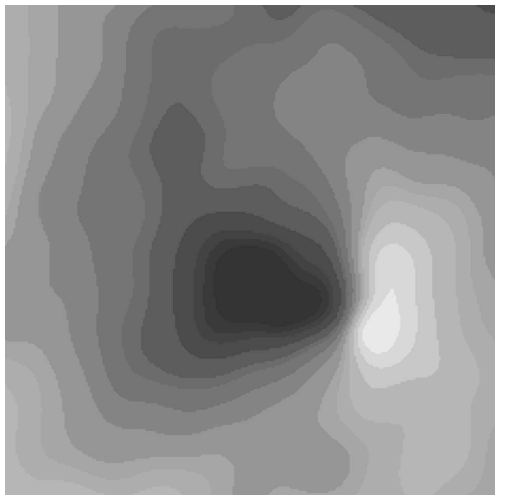

c)

Fig. 2. Mask size reduction effect on atomic scale noise. $b$ and $c$ are the phase images for a same component of $a$, the mask size are $g / 2$ and $g / 6$, respectively.

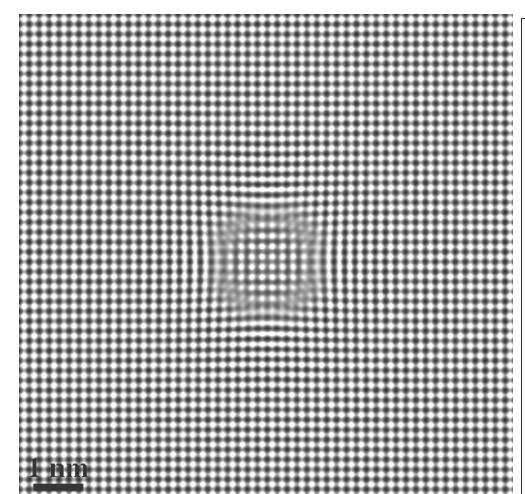

a)

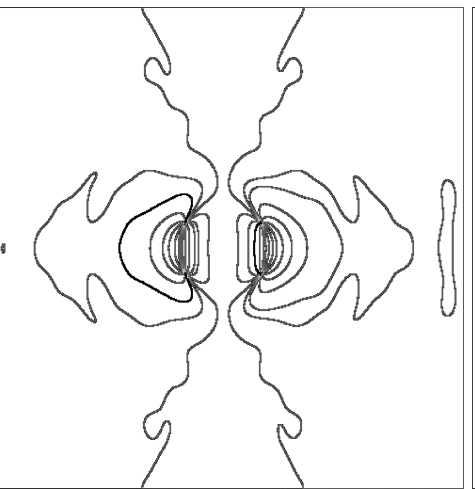

b)

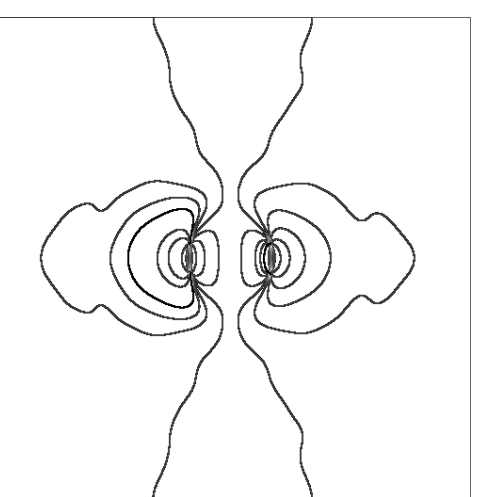

c)

Fig. 3. Effect of mask size reduction according to simulated image. a is a simulated HREM image of a precipitate embedded in a matrix, $b$ and $c$ show contour maps of the phase images obtained with a mask size $g / 2$ and $g / 6$ respectively. 
The areas necessary to draw the strain and stress fields and further study the dislocation propagation must be large and finely sampled. Here the adequate conditions are $2048 \times 2048$ pixels $^{2}$ with $0.02 \mathrm{~nm}$ per pixel. In spite of the rapid development of CCD cameras for image acquisition, nowadays HREM microscopes rarely fulfill these requirements. Therefore digitizing of the HREM images is frequently done with scanners. Unfortunately, the scanner but also the emulsion on negative may be responsible for fluctuations of the image intensity.

The question is then whether long-range intensity fluctuations on the digitized images can alter measurements. Long range fluctuations forming an additive contribution should not affect the phase image because the Fourier transform of a long-range fluctuating function is narrow and centered on the origin and thus has no contribution close to the selected $\mathrm{g}$ vector. Therefore, in the reciprocal space, the area taken by the mask is not affected by an additive contribution. This property can be easily tested using a clean image in which some long-range intensity fluctuations were introduced either by addition or by multiplication. The HREM image in Fig. 1a was noticeably modified by the addition of a grey level ramp (Fig. 4a) and an inhomogeneous fluctuation derived from an experimental HREM image (Fig. 4b).

The operation was also done using multiplication instead of addition. In case of addition of long-range fluctuations, Fig. 5a shows that we obtain the same phase images as with the original image (Fig. 1b). As illustrated by Fig. 5b when compared to Fig. 5a or 1b, in case of multiplicative background, the phase image is quite altered. By subtraction of phase images, we estimate the effect to about $10 \%$. So the effect of multiplicative background is small but not negligible. It comes from the fact that a multiplication in direct space corresponds to a convolution in reciprocal space. Therefore only very long-range fluctuations would have no effect in case of multiplication.

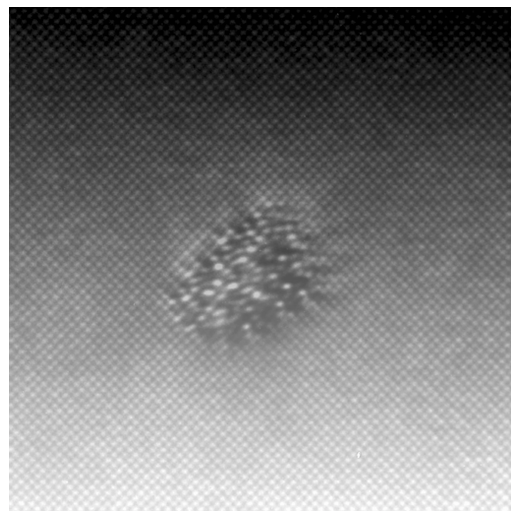

a)

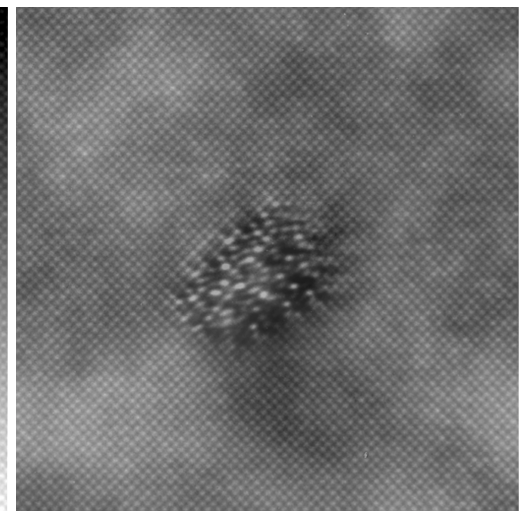

b)

Fig. 4. Addition of long range intensity fluctuation to an experimental HREM image. a and $b$ attest of the strong level of fluctuation introduced, for comparison see the original image in Fig. Ia. In Fig. $4 a$ and $b$ the background is added to the original image.

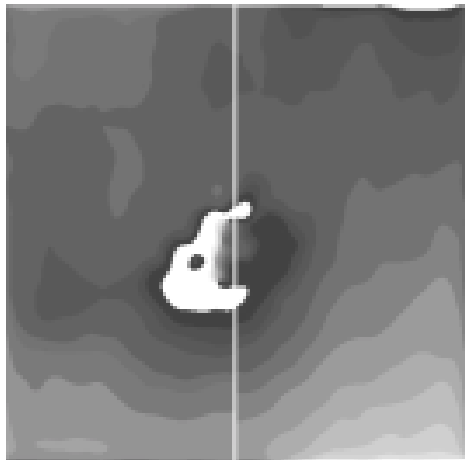

a)

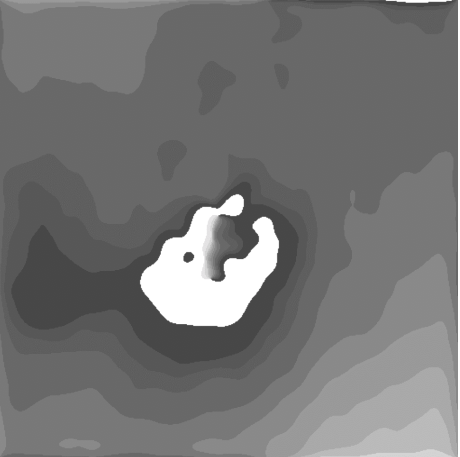

b)

Fig. 5. Phase images after background introduction. a corresponds to two semi images derived from Fig. $4 a$ and $4 b$. Notice that these phase images are identical to Fig. $1 b . b$ is the phase image obtained with $a$ multiplicative background instead of a additive one. Comparison with Fig. 1 in indicates a stronger effect. 
This illustration of the impact of an additive or multiplicative contribution points out the need to identify the type of long-range intensity fluctuations. In that purpose, a simple solution consists in applying a rolling ball operation and background subtraction on the HREM images. If, after such operation, the image intensity is constant and the amplitude of the lattice fringes unchanged, it means that the long-range fluctuations form an additive contribution. Then the phase image method can be applied to the original HREM image. An alternative is to compare the phase images before and after background subtraction, if they are unchanged, it means that the long-range intensity fluctuations result in additive background.

\section{HREM IMAGE SPECIFIC ABERRATION}

For HREM imaging, the electron beam is focussed by electromagnetic lenses. Therefore the lens aberration may affect the images. In particular, the astigmatism aberration, which is only partly corrected by dedicated stigmators, can generate artifacts. Depending on the microscope, a so-called "3-fold astigmatism" may give rise to a more or less pronounced rigid body translation (Krivanek et al., 1995). In our case, such translation would be interpreted as a displacement. Hopefully, the 3-fold astigmatism effect can be calculated and introduced in image simulation. HREM simulated images of a precipitate such as the one in Fig. 3a were calculated with and without 3-fold astigmatism. Consistently with (Krivanek et al., 1995), phase image comparison shows that the artifact is limited to the precipitate. Since our point is ultimately to study the dislocation motion in the matrix and approaching the precipitate but not at the matrix/precipitate interface, the 3-fold astigmatism artifact is not a problem here. Besides, this effect can be reduced by the choice of the microscope.

\section{DISTORTION DUE TO SCANNERS}

Unfortunately scanners also are possible sources of distortion. This can be tested with gratings and HREM images of perfect lattices. According to the performed Fourier transforms, in our work conditions, the distortion of a square is smaller than $0.07 \%$ with a scanner of negatives. Indeed, such distortion gives no artifact on phase image because it is taken into account in the phase image calculation. Nevertheless, phase images have pointed out some scanner defects illustrated by Fig. 6, which shows the phase image of a same area digitized under different conditions. Notice that these scanner defects are all the more pronounced that the area to digitize is large. On the phase image, major features are preserved but there is an obvious scanner contribution (amplitude approximately 0.01 $\mathrm{nm})$. Such effect prevents from looking at very small displacements. Hopefully this digitizing problem should be temporary since, in the future, HREM microscopes will be equipped with large CCD camera. If distortions are still detected, correction of the CCD response will be then possible.

\section{SIMULATION OF DISLOCATION MOTION IN THE STRAIN FIELD}

To study the influence of precipitation on the mechanical properties of the material we have simulated the movement of dislocations within the stress field determined from the measured displacement field. The simulation uses the equations of the dynamics of dislocations, including short range and long range interactions and allows for any dislocation character. For instance, we have calculated the motion of a dislocation in the experimental strain field obtained using the phase image, several steps are illustrated in Fig. 7.

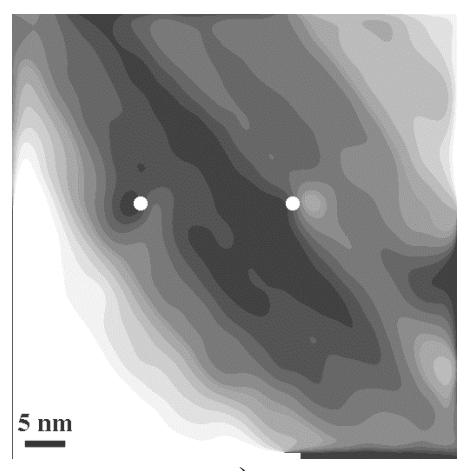

a)

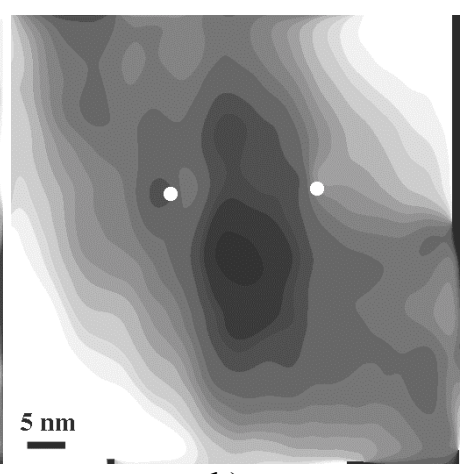

b)

Fig. 6. Phase images of a same area with two precipitates (here marked by white dots). The HREM area has been digitized in different conditions: in order to point out scanner effect, the image has been rotated in different ways before digitizing. We observe a distortion following the scanning direction. 


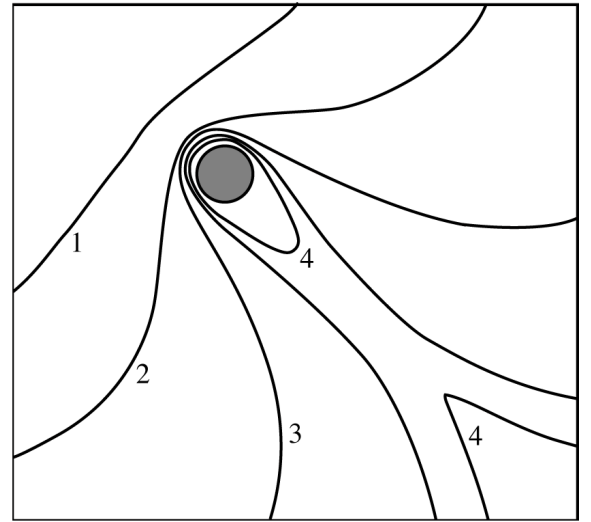

Fig. 7. Simulation of the dislocation propagation in the experimental strain field. Lines labeled 1 to 4 give several positions of the dislocation line during propagation. According to the simulation which only takes on the precipitates only the strain field they create in the matrix, the dislocation by-pass the precipitates resulting in the formation of an Orowan loop.

In the Al-6056 T6 case, the simulation indicates that for an applied stress close to the yield stress, dislocation motion in the material occurs by circumventing of precipitates through activation of the Orowan process. This prediction was confirmed by observations of $2 \%$ deformed samples by transmission electron microscopy. Experiments showed that the Orowan process mainly occurred during deformation and that only a very few precipitates are sheared, in good accordance with our simulations.

Simulations of dislocation propagation in the experimental strain field are still under progress. We have in particular to estimate the impact of scanner distortion that cannot be eliminated. However, the next step will be to carry out phase images on HREM images digitized on line with CCD camera.

\section{ACKNOWLEDGEMENT}

This work has been supported by the CNRS "Programme Matériaux" projet n¹96.

\section{REFERENCES}

Douin J, Donnadieu P, Epicier T, Dirras GF, Proult A, Silvain JF (2001). Stress field around precipitates: direct measurement and relation with the behavior of dislocations. Proceeding ICSMA-12, to appear in Material Science Engineering A.

Mott NF, Nabarro FRN (1940). Proc Phys Soc 52:86.

Hytch MJ, Snoeck E, Kilaas R (1998). Quantitative measurements of displacement and strain fields from HREM micrographs. Ultramicroscopy 74:131-46.

Krivanek OL, Stadelmann PA (1995). Effect of the threefold astigmatism on high resolution electron micrographs. Ultramicroscopy 60:103-13. 